\title{
Multiple charge beam dynamics in alternate phase focusing structure
}

\author{
S. Dechoudhury, ${ }^{1, *}$ Alok Chakrabarti, ${ }^{1}$ and Y.-C. Chao ${ }^{2}$ \\ ${ }^{1}$ Variable Energy Cyclotron Centre, I/AF Bidhan Nagar, Kolkata 700 064, India \\ ${ }^{2}$ TRIUMF, 4004 Wesbrook Mall, Vancouver, British Columbia, Canada V6T 2A3
}

(Received 19 March 2014; published 15 July 2014)

\begin{abstract}
Asymmetrical alternate phase (A-APF) focusing realized in a sequence of 36 superconducting quarter wave resonators (QWRs) has been shown to accelerate almost $81 \%$ of input uranium beam before foil stripper to an energy of $6.2 \mathrm{MeV} / \mathrm{u}$ from $1.3 \mathrm{MeV} / \mathrm{u}$. Ten charge states from $34+$ to $43+$ could be simultaneously accelerated with the phase of resonators tuned for $34+$. The A-APF structure showed the unique nature of a large potential bucket for charge states higher than that of the tuned one. Steering inherent to QWRs can be mitigated by selecting appropriate phase variation of the APF periods and optimization of solenoid field strengths placed in each of the periods. This mitigation facilitates the multiple charge state acceleration scheme.
\end{abstract}

DOI: 10.1103/PhysRevSTAB.17.074201

PACS numbers: 29.27.Bd

\section{INTRODUCTION}

Superconducting quarter wave resonators (QWRs) are widely used for the acceleration of stable as well as rare heavy-ion beams. In isotope seperator on-line (ISOL)-type rare ion beam (RIB) facilities employing linear accelerators for RIB acceleration, superconducting QWR cavities are often used to accelerate the beam to energies enough to carry out Coulomb barrier physics, usually in the range 5 to $7 \mathrm{MeV} / \mathrm{u}$, after initial acceleration in radio frequency quadrupole (RFQ) and room temperature linacs up to energy of about $1 \mathrm{MeV} / \mathrm{u}$. TRIUMF's ISAC II facility [1] is a typical example where this acceleration scheme has been implemented successfully. For RIB acceleration, the transmission efficiency of the accelerating structure is one of the most important considerations since one cannot afford to lose the beam intensity in the process of acceleration. Superconducting QWR (SC QWR) cavities with independent phase and high field levels can accept heavyion beams with appreciable transverse and longitudinal emittances and can thus accelerate input beams coming out of the room temperature accelerators of the preceding acceleration stages with practically no loss in the beam intensity. However, to optimally use the high accelerating field of SC QWRs a charge stripper is used upstream of the SC QWR accelerators to increase the charge state of the unstable heavy-ion beam. This scheme is equally suitable for acceleration of high current uranium beams to very high energies since it is difficult to get enough intensity for high charge states from the ion source, for example from an

\footnotetext{
*sdc@vecc.gov.in

Published by the American Physical Society under the terms of the Creative Commons Attribution 3.0 License. Further distribution of this work must maintain attribution to the author(s) and the published article's title, journal citation, and DOI.
}

electron cyclotron resonance (ECR) ion source. It is often advantageous to extract comparatively low charge state and higher intensity uranium beam from the ion source, accelerate it to about $1 \mathrm{MeV} / \mathrm{u}$ and increase the charge state by stripping before accelerating further in SC QWRs. The stripper, however, has an undesirable feature. It produces a number of charge states [2] and one chooses the most abundant one for further acceleration through the QWRs. A major fraction of the beam is thus lost in the process. One can gain manifold in beam intensity if multiple charge states can be simultaneously accelerated. Numerical simulation of a driver linac for rare ion beam facility accelerating multiple charge states of uranium beam after stripper has been carried out earlier [3]. Simulation showed that five charge states centered around $q=75+$ can be simultaneously accelerated from 12.3 to $85.5 \mathrm{MeV} / \mathrm{u}$. Also, eight charge states of uranium beam had been simultaneously accelerated from $286 \mathrm{MeV}(1.2 \mathrm{MeV} / \mathrm{u})$ to $690 \mathrm{MeV}(2.9 \mathrm{MeV} / \mathrm{u})$ through a section of ATLAS [4]. In this case simultaneous acceleration of multiple charge states has been achieved through comparatively low acceleration gradient; about $400 \mathrm{MeV}$ total energy gain in 24 split coaxial SC resonators.

In the case considered in Ref. [3], the stripping was considered at higher energy producing an equilibrium charge state of $q=75+$ for uranium. This has an advantage. Owing to high charge to mass ratio the phase offset for other charge states is small when resonators had been tuned for $q=75+$. Also the width of charge state distribution is comparatively small at higher energy $(\sim 12 \mathrm{MeV} / \mathrm{u})$. The situation is much more complicated for stripping at lower energy (say $1.3 \mathrm{MeV} / \mathrm{u}$ ), since stripping results in a much broader charge distribution around the equilibrium charge state. As an example, uranium beam stripped at $1.3 \mathrm{MeV} / \mathrm{u}$ is distributed over 12 charge states from $32+$ to $43+$ around the equilibrium charge of $37+$. In such cases, 


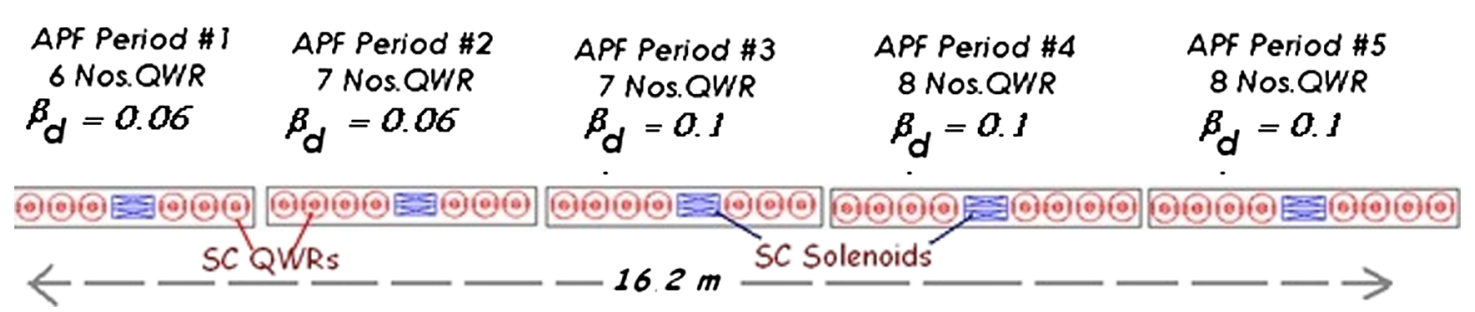

FIG. 1. Layout showing the configuration of cryomodules consisting of SC QWRs and solenoids.

simultaneous acceleration of multiple charge states through a long chain of independently phased resonators without compromising the accelerating gradient becomes challenging and difficult. The asymmetrical alternate phase focusing (A-APF) $Q W R$ structure [5,6,7], owing to its inherent larger longitudinal and transverse acceptances, seems to be the only candidate that can in principle provide a solution for multiple charge acceleration. It has been shown earlier that by suitably changing the sign of the synchronous phase longitudinal and transverse beam focusing can be realized over a long chain of QWRs [7]. Detailed theoretical analysis corroborated by particle tracking code showed [7] that the A-APF scheme can provide appreciable longitudinal and transverse acceptance area and this design has been proposed for the ANURIB facility at VECC [8].

Acceptance of multiple charge acceleration depends on the effective focusing system which can limit the transverse emittance growth [4]. Also, the longitudinal dynamics becomes extremely challenging for simultaneous multicharge acceleration if one wants to keep a good enough acceleration gradient. A-APF, as brought out in our earlier work [7], provides an effective transverse and longitudinal focusing without the need for a separate longitudinal (rebuncher) and transverse (solenoids) focusing device even without compromising the acceleration gradient. In case of quarter wave resonators (QWR) steering effect due to the asymmetric field profile would lead to effective emittance growth. It has already been shown [7] that steering effects gets mitigated over a period due to the oscillating phase profile of the A-APF configuration thus limiting the transverse emittance growth facilitating the possibility of multiple charge acceleration. This prompted us to look into the efficacy of A-APF systems in multiple charge acceleration especially with QWRs as the accelerating cavity.

The present paper addresses the design of a multiple charge acceleration scheme through such an A-APF structure realized in a sequence of 36 quarter wave resonators. Changes have been incorporated on the base line design of the earlier A-APF scheme [7] in order to facilitate the option of multiple charge state acceleration. Steering kicks that depend on the energy of the beam and phase of resonator [9] have been found to play a dominant role in case of simultaneous acceleration of multiple charge states.
Accordingly, optimization of phase and solenoid fields has been carried out in each cryomodule, which could ensure a common transverse and longitudinal acceptance for most of the post-stripper charge states. The final acceleration scheme consisting of 36 QWRs with $\beta_{\mathrm{d}}$ of 0.06 and 0.1 is shown in Fig. 1. Using this set of QWRs operated in A-APF mode, 10 charge states (from $34+$ to $43+$ ) of uranium beam could be accelerated efficiently from 1.3 to $6.2 \mathrm{MeV} / \mathrm{u}(\sim 1160 \mathrm{MeV}$ total energy gain in 36 resonators).

\section{DESIGN OF A-APF CONFIGURATION}

\section{A. Potential bucket for different charge states}

The designed beta $\left(\beta_{\mathrm{d}}\right)$ for QWR's is selected on the basis of transit time factor over the energy range. We have chosen frequency to be $100 \mathrm{MHz}$, aperture diameter $20 \mathrm{~mm}$ and gap to $\beta_{\mathrm{d}} \lambda$ ratio as 0.2 . The choice of designed beta for the resonators $\left(\beta_{\mathrm{d}}\right)$ as well as the number of resonators in a period takes care of the fact that transit time factor (TTF) remains greater than 0.8 for the entire energy range. In a particular period, the set of resonators has synchronous phase obeying a stepwise function. Using stability analysis the phases and electric field of the resonators in APF periods have been chosen so that cosine of transverse and longitudinal phase advance lies close to 0 . This ensured a strong longitudinal and transverse focusing in each such APF period. Detailed analysis to ascertain these choices has already been covered in Ref. [7]. The present design exploits this particular feature of strong focusing in both dimensions for achieving multiple charge state acceleration.

The phase acceptance and energy width of any particular charge state can be derived from an effective potential [10]. The effective potential in the present paper has been calculated using smooth approximation formalism with acceleration [11]. The on-axis electric fields for these calculations [7] have been simulated using CST [12]. In order to analyze the efficacy of smooth approximation with acceleration in predicting the phase acceptance of the periods, we had carried out particle tracking with real 3D fields of the cavities. Retracing back the successful particles the phase acceptance was determined. The phase acceptance values were found to be within $\pm 5 \%$ of the 
value predicted by the smooth approximation formalism [7]. The resonator phases have been optimized as per the A-APF scheme for a particular charge state to be termed as the "tuned" charge state. Once the potential bucket has been calculated for the tuned charge state, one can derive the buckets for other charge states with the same phase tuning using the velocity and time profile for other charge states.

The calculations show that the potential bucket becomes shallower (decrease in energy width acceptance) for charge states lower than the tuned one. On the other hand, for higher charge states appreciable energy and phase width still exist. So, one can in principle accelerate charge states higher than that of the tuned one. The potential buckets for different charge states of uranium for the first focusing period comprised of six QWRs are shown in Fig. 2 when the resonators are tuned for $q=34+$. It is evident that for charge states higher than $q=34+$, the depth of the effective potential increases with the charge state. However, there is a flip side-there occurs a shift in the synchronous phase (for which energy width acceptance is maximum) for higher charge states. This effect is not appreciable for a single period (or a number of resonators) but becomes significant with increase in the number of resonators, decreasing thereby the common acceptance area amongst different charge states. The choice of tuned charge state less than 34+ would lead to inefficient acceleration of dominantly produced charge states after the stripper.

\section{B. Steering effect and optimization of solenoids}

In quarter wave resonators (QWR) owing to its resonant structure, the electric and magnetic fields in the accelerating gaps are not axially symmetric. QWRs have in addition to accelerating field (along z), a vertical electric field (Ey) and a horizontal magnetic field $(\mathrm{Bx})$. In case of superconducting cavities, due to high acceleration gradients, both the fields have prominent steering effects on the beam. Steering

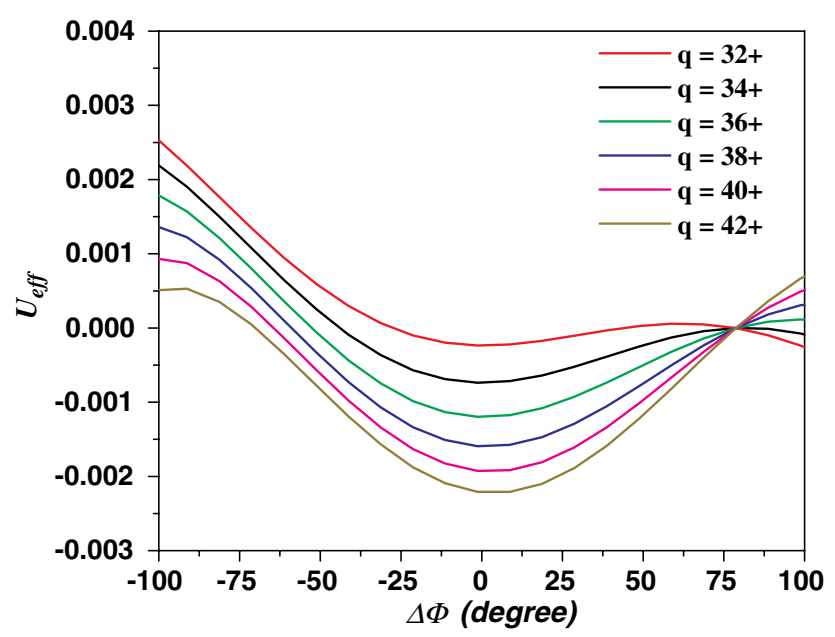

FIG. 2. Potential bucket calculated for different charge states for APF period 1 tuned for $q=34+$. depends on the resonator's synchronous phase, beam velocity and acceleration gradient [9]. The problem of steering becomes more dominant in the case of multiple charge state acceleration, as different charge states have different velocity profiles. It reduces the acceptance area and shifts the longitudinal acceptance phase space from that of the tuned one.

Steering being dependent on sinusoid of phases, it changes sign with the sign of individual resonator phases. In a particular APF period as a sign of phase oscillates from positive to negative the steering kick gets canceled [7]. Similarly, if the last QWR of an APF period and the first QWR of the immediate next period have the same phase sign, steering kick gets amplified in the same direction, reducing the common acceptance area for multiple charge state acceleration. Accordingly, one needs to change the A-APF scheme that has been studied in our earlier paper [7] to facilitate the option of multiple charge state acceleration. We have considered alternating positive and negative cycle of variation in phase, when going from one period to the next as shown in Fig. 3. This reduces the steering effect to a larger extent. The selected electric field gradient (product of electric field and TTF) for the cavities in five cryomodules are $5.3,5.2,4.1,3.5$ and $3.8 \mathrm{MV} / \mathrm{m}$, respectively.

The $Y$ direction steering can be calculated using the electric and magnetic field profile as seen by the particle while traversing through the resonators. The field integration formula for steering described in Ref. [13] has been utilized to calculate the steering for different charge states. Solenoids placed in each cryomodule produce $X-Y$ coupling as well as focusing of the beam. Steering caused by QWRs before the solenoids would shift the particle to offaxis trajectory and after solenoid it would enter the next set of QWRs with the combined effect of solenoids and steering. It has been found that $X-Y$ coupling results in transferring a part of $Y$ direction kick to $X$. Thus solenoids

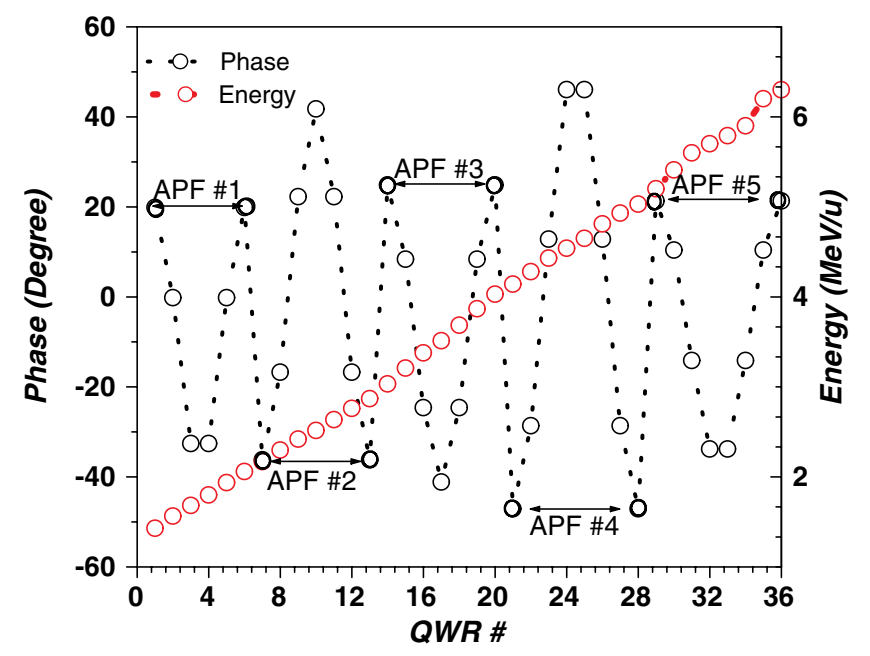

FIG. 3. Alternating phase variation and energy profile along the APF periods. 

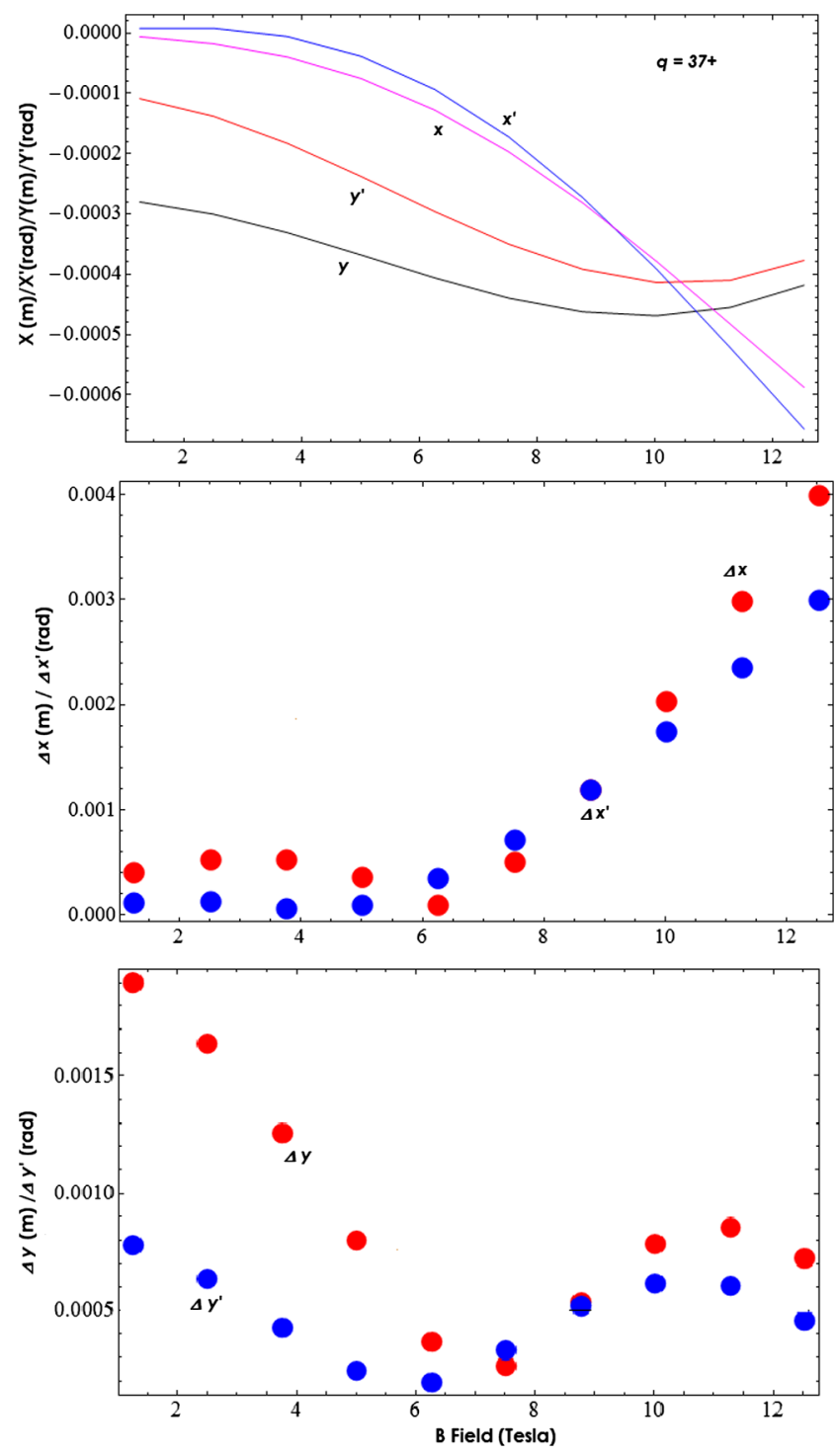

FIG. 4. Variation of beam coordinates and divergence of $37+$ and range spanned by different charge states at end of APF\#2 with solenoid field.

could be tuned within an optimum range so that different on-axis charge states can be close to the axis and have lesser divergence as it exits the period. The coupled equation of motion for solenoids has been described in Ref. [14]. Using MATHEMATICA [15], equations of motion have been solved for different charge states to find out the transverse coordinates and divergence at the end of each period taking into the effect of solenoid focusing and steering in the cavities. Figure 4 depicts variation of coordinate and divergence at the end of the second focusing period for $q=37+$ and width of distribution in transverse coordinates for different charge states.

The solenoids in each focusing periods have been optimized so that most dominant charge state $37+$ on-axis particle undergoes minimum $Y$ steering at the end of each APF period. It was also ensured that different charge states (having different velocity profiles through resonators) lie within the close proximity of the dominant charge state at the end of the periods. Such optimization also ensured minimum transverse emittance growth for all charge states combined as compared to single charge acceleration. The solenoids considered here are of length $0.25 \mathrm{~m}$ and for maximum optimized field strength of $9 \mathrm{~T}$, the field at the nearest QWR (end of preceding QWR and start of succeeding QWR) is only around 120 Gauss which is much below the critical magnetic field of $\mathrm{Nb}$.

\section{BEAM DYNAMICS SIMULATION}

\section{A. Particle tracking simulation}

The particle tracking has been carried out using GPT [16] with 3D fields of two types $\left(\beta_{\mathrm{d}}=0.06\right.$ and 0.1$)$ of QWRs obtained from CST simulation. Space charge is not considered since for applications considered here the beam intensity is never high enough for the space charge to play a significant role. An aperture of $10 \mathrm{~mm}$ has been considered in GPT for particle tracking through all the resonators and solenoids. An optimization algorithm as described in the previous section dictated the values of five solenoids. Beam having distribution in the $Y-Y^{\prime}$ plane and $\Delta \mathrm{E}-\Delta \phi$ with $X=X^{\prime}=0$ has been tracked for charge states from $34+$ to $42+$ in order to find common longitudinal acceptance. Gaussian distribution with normalized longitudinal emittance of $4 \pi \mathrm{keV} / \mathrm{u}$ nsec (phase rms width $\sim \pm 2 \mathrm{deg}$ and energy rms width $\sim \pm .01 \mathrm{MeV} / \mathrm{u}$ ) with the orientation the same as that of acceptance ellipse, and uniform distribution of transverse emittance (both in $X$ and $Y$ ) $0.2 \pi \mathrm{mmmrad}$ have been generated using MATHEMATICA, as the input beam. These values are nearly equal to those measured for the ISAC I output beam at TRIUMF [17].

\section{B. Multiple charge state acceleration}

Separate particle tracking for each charge state from $34+$ to $42+$ has been carried out with the set of input particle distribution created. With distribution in $X$ and $X^{\prime}$ fine-tuning of solenoids close to the optimized values has been done to maximize the transmission efficiency for the dominant charge state $q=37+$, by an iterative method. The optimized solenoid field in each cryomodule is $6.8,6$, 7.5, 6.0, and $9 \mathrm{~T}$ respectively.

For $q=34+$, only $47 \%$ are accelerated to energy more than $6 \mathrm{MeV} / \mathrm{u}$, while for higher charge states almost all particles reaching the end are accelerated to energy in excess of $6 \mathrm{MeV} / \mathrm{u}$. A detailed curve showing the transmission efficiency along with the charge state fraction of all the charges created after stripping is shown in Fig. 5.

Considering the fraction with which a particular charge state is created after stripping at $1.3 \mathrm{MeV} / \mathrm{u}$ and the fraction of it accelerated to energy greater than $6.0 \mathrm{MeV} / \mathrm{u}, 81.5 \%$ of the input uranium beam before 


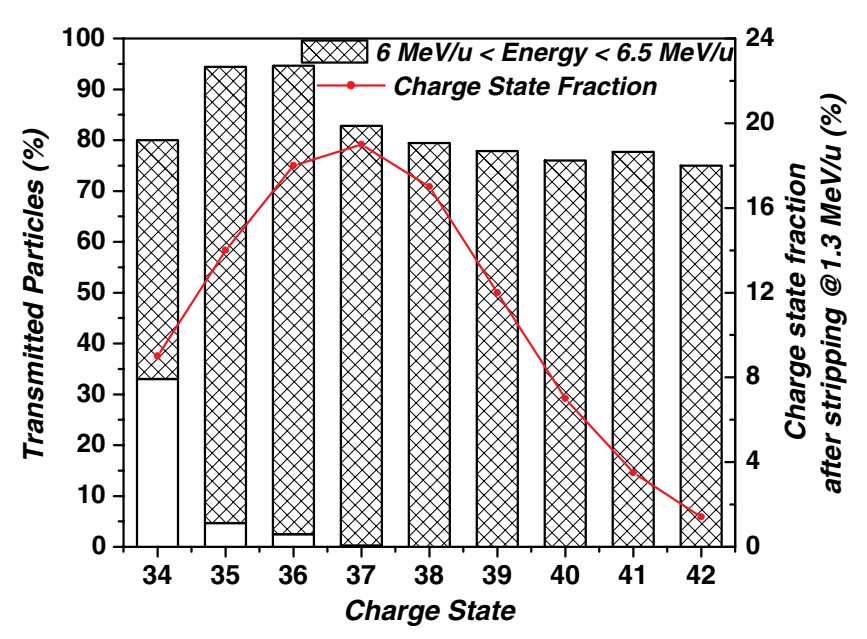

FIG. 5. Transmission efficiency of multiple charge state.

the foil stripper has been finally accelerated to $6.23 \mathrm{MeV} / \mathrm{u}$ with FWHM of $\pm 1.5 \%$. In this configuration $73 \%$ of $q=$ $43+$ beam can also be accelerated to $6.29 \mathrm{MeV} / \mathrm{u}$ with FWHM of $\pm 0.5 \%$ although its charge state fraction is less than $1 \%$. Final transverse and longitudinal distribution of all the charge states at $0.3 \mathrm{~m}$ after the last QWR of the fifth period is shown in Fig. 6. These represents only the fraction that has been accelerated to energy of $6 \mathrm{MeV} / \mathrm{u}$ or more.
TABLE I. Normalized emittance and energy values for different charge states.

\begin{tabular}{llcccl}
\hline \hline & $\begin{array}{c}\text { Normalized } \\
\text { rms EmitX } \\
(\pi \mathrm{mm} \text { mrad })\end{array}$ & $\begin{array}{c}\text { Normalized } \\
\text { rms EmitY } \\
(\pi \mathrm{mm} \text { mrad })\end{array}$ & $\begin{array}{c}\text { Normalized rms } \\
\text { EmitZ } \\
(\pi \mathrm{keV} / \mathrm{u} \mathrm{nsec})\end{array}$ & $\begin{array}{c}\text { Mean } \\
\text { energy } \\
(\mathrm{MeV} / \mathrm{u})\end{array}$ \\
\hline $34+$ & 0.1874 & 0.18966 & 0.00383 & 6.37 & 0.055 \\
$\mathrm{u}$ & $\mathrm{MEV}$ \\
$35+$ & 0.1732 & 0.1588 & 0.00491 & 6.28 & 0.103 \\
$36+$ & 0.269 & 0.19378 & 0.00729 & 6.29 & 0.12 \\
$37+$ & 0.259 & 0.1874 & 0.00225 & 6.27 & 0.08 \\
$38+$ & 0.245 & 0.1394 & $3.77 \times 10^{-4}$ & 6.24 & 0.02 \\
$39+$ & 0.1757 & 0.1261 & $2.46 \times 10^{-4}$ & 6.2 & 0.017 \\
$40+$ & 0.1709 & 0.1039 & $2 \times 10^{-4}$ & 6.18 & 0.025 \\
$41+$ & 0.12833 & 0.09669 & $1.8 \times 10^{-4}$ & 6.17 & 0.03 \\
$42+$ & 0.1138 & 0.10626 & $1.79 \times 10^{-4}$ & 6.13 & 0.02 \\
\hline \hline
\end{tabular}

Emittance values along with mean energy of different charge states have been tabulated in Table I. The transmission efficiency corresponding to different mismatched longitudinal input beam (Fig. 7) shows an appreciable tolerance factor of $\sim 20 \%$ in input beam.

Misalignment of resonators and solenoids would also have an adverse effect on transverse beam dynamics and hence on beam transmission efficiency. In an earlier paper [3] transverse misalignment considered for solenoids was $\pm 100 \mu \mathrm{m}$. GPT simulation carried out for the present design, using 5000 particles distributed equally amongst
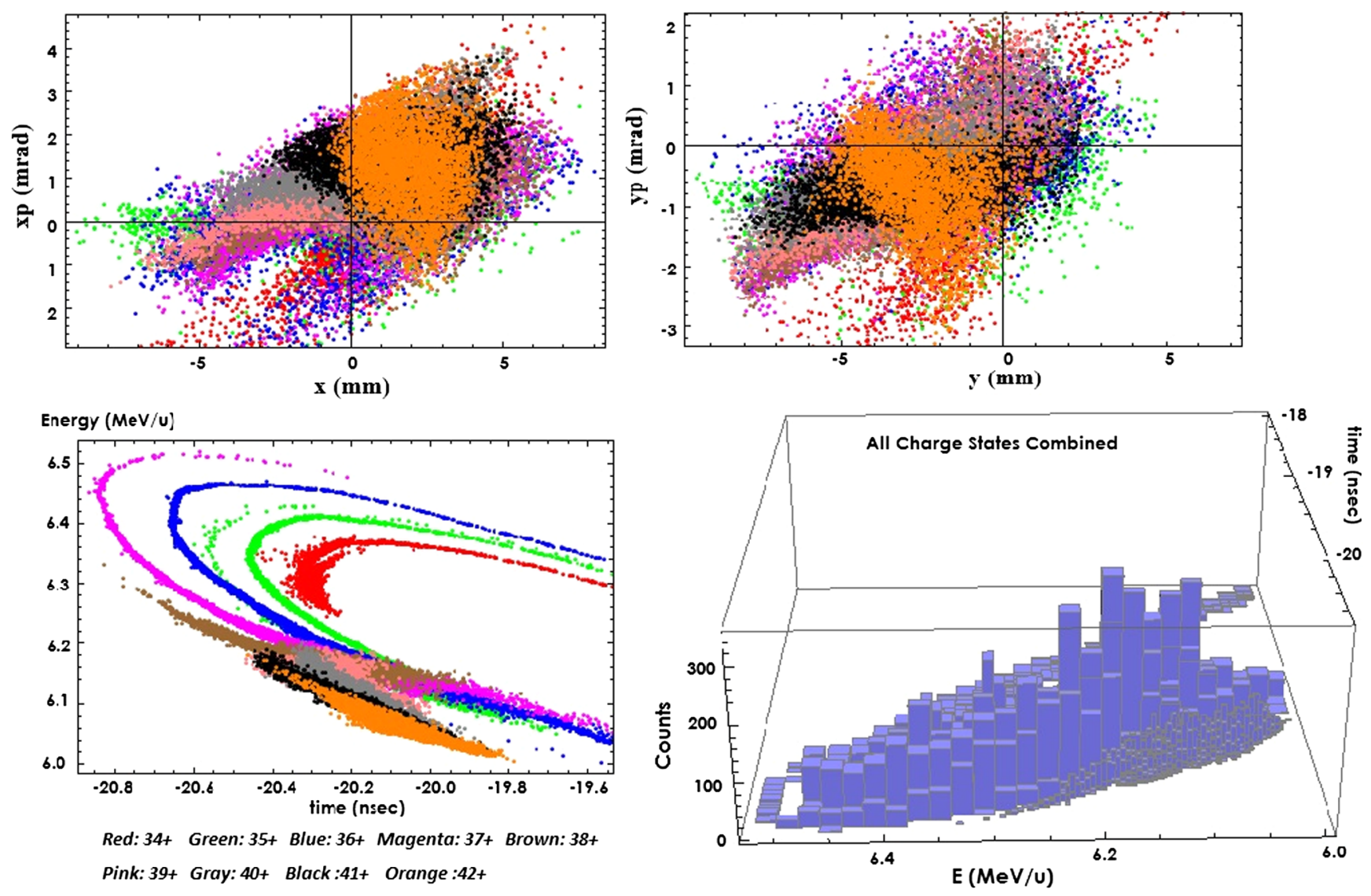

FIG. 6. Final distribution of nine charge states from $34+$ to $42+$ accelerated to $6.2 \mathrm{MeV} / \mathrm{u}$. 


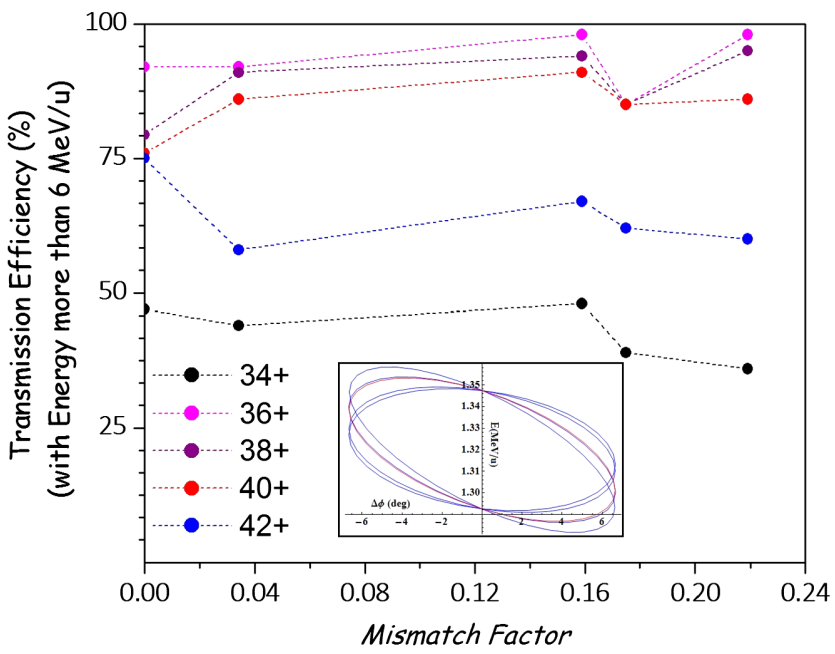

FIG. 7. Transmission efficiency with mismatch factor for different charge states. The inset shows the orientation of the mismatched ellipse (red showing the acceptance ellipse).
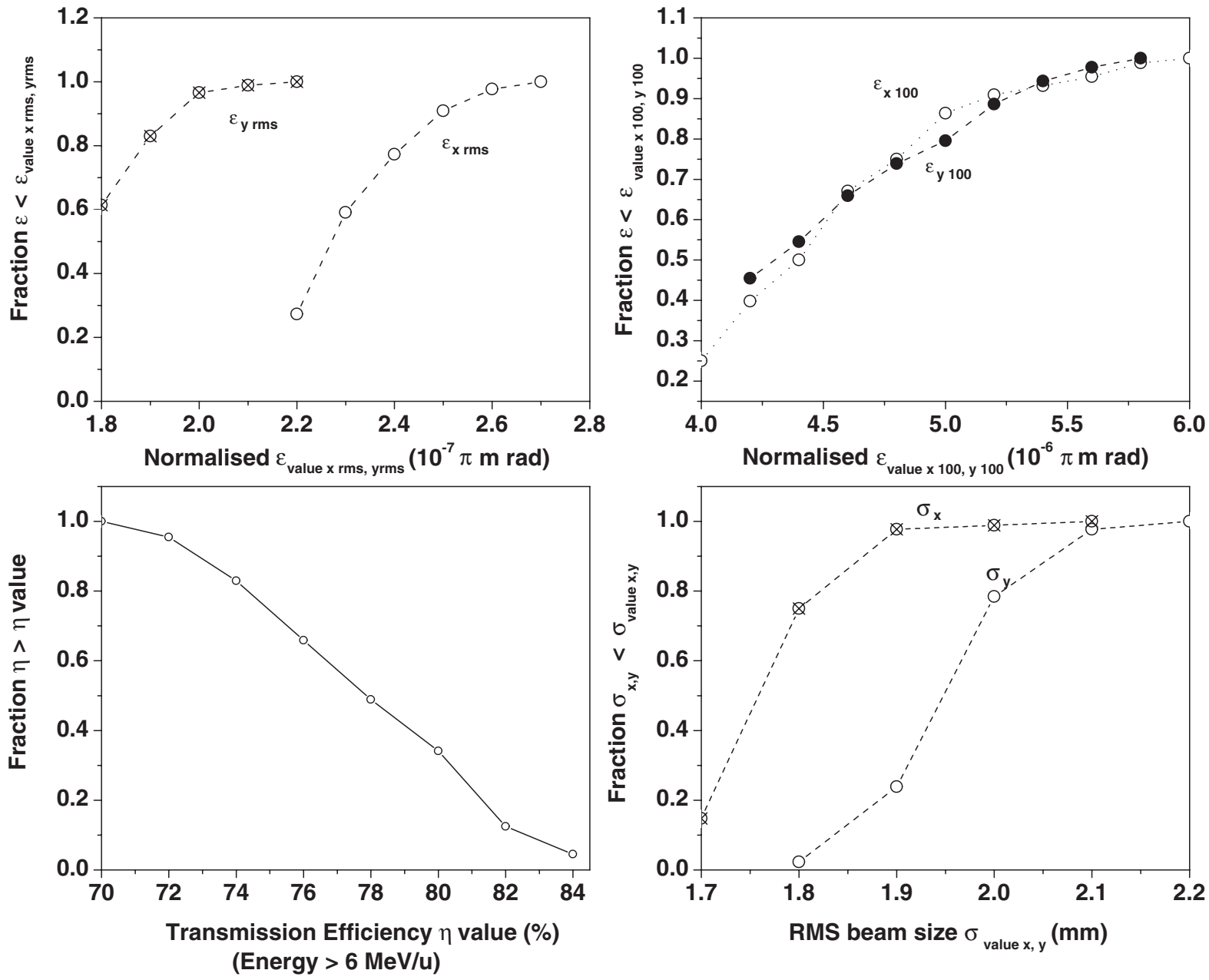

FIG. 8. Probability distribution for transmission efficiency (energy $>6 \mathrm{MeV} / \mathrm{u}$ ), transverse emittance and rms beam size. 


\section{CONCLUSION}

It is shown that a A-APF structure can be used to accelerate with high efficiency multiple charge states simultaneously through a long chain of quarter wave resonators (QWRs) maintaining a high enough acceleration gradient. APF structure showed the unique nature of the large potential bucket for charge states higher than that of the tuned one resulting in good enough longitudinal acceptance for different charge states. Steering inherent to QWRs limits the longitudinal and transverse acceptance of all charge states posing a serious problem. However, this effect can be mitigated by selecting appropriate phase variation of APF periods and optimizing solenoids placed in each of the periods. In this design, one solenoid per cryomodule has been found to be enough to transmit the beam with $81 \%$ efficiency while it got accelerated from 1.3 to $\sim 6.2 \mathrm{MeV} / \mathrm{u}$. Increasing the number of solenoids may help marginally to increase the efficiency but this would increase the complexity of the cryomodule and of course the cost. Present study showed A-APF as a viable and potential candidate for such multiple charge state acceleration starting from charge stripping at low energy $\sim 1 \mathrm{MeV} / \mathrm{u}$ (inducing larger charge state distribution width) and accelerating to such higher energy $\sim 6.2 \mathrm{MeV} / \mathrm{u}$ without compromising acceleration gradient.

\section{ACKNOWLEDGMENTS}

This work is a part of the research project funded by the Department of Atomic Energy for the development of ANURIB Facility.
[1] R. E. Laxdal, in Proceedings of the 21st International Linac Conference, Gyeongju, Korea, 2002 (Pohang Accelerator Laboratory, Pohang, Korea, 2002).

[2] K. Shima, N. Kuno, M. Yamanouchi, and H. Tawara, At. Data Nucl. Data Tables 51, 173 (1992).

[3] P. N. Ostroumov and K. W. Shepard, Phys. Rev. ST Accel. Beams 3, 030101 (2000).

[4] P. N. Ostroumov, R. Pardo, G. Zinkann, K. Shepard, and J. Nolen, Phys. Rev. Lett. 86, 2798 (2001).

[5] V. V. Kushin, in Linear Ion Accelerators, edited by B. P. Murin (1978), Chap. 7, p. 173.

[6] V. V. Kapin and A. Nesterovich, in 22nd Proceedings of RuPAC, Russia, 2010 (Institute of High Energy Physics, Protvino, Russia, 2010).

[7] S. Dechoudhury and A. Chakrabarti, Phys. Rev. ST Accel. Beams 16, 052001 (2013).

[8] A. Chakrabarti, A. Bandyopadhyay, V. Naik, S. Dechoudhury, M. Mondal, and P. Y. Nabhiraj, Nucl. Instrum. Methods Phys. Res., Sect. B 317, 253 (2013).

[9] A. Facco and V. Zvyaginstev, Phys. Rev. ST Accel. Beams 14, 070101 (2011).

[10] H. Okamoto, Nucl. Instrum. Methods Phys. Res., Sect. A 284, 233 (1989).

[11] J. Qiang and R. W. Garnett, Nucl. Instrum. Methods Phys. Res., Sect. A 496, 33 (2003).

[12] Computer Simulation Technology, Darmstadt, Germany, website: http://www.cst.com.

[13] P. N. Ostroumov and K. W. Shepherd, Phys. Rev. ST Accel. Beams 4, 110101 (2001).

[14] M. Reiser, Theory and Design of Charged Particles (Wiley-VCH Verlag $\mathrm{GmbH} \& \mathrm{Co}$. KGaA, Weinheim, 2008), 2nd ed., p. 67.

[15] Wolfram Research, Inc., MATHEMATICA, Version 7.0, Champaign, IL (2008).

[16] General Particle Tracer, website: http://www.pulsar.nl/gpt.

[17] M. Marchetto (private communication).

[18] J. E. Stovall et al., in Proceedings of 23rd LINAC Conference, Geneva, Switzerland, 1996. 